\title{
PARÂMETROS DE EROSIVIDADE DA CHUVA E DA ENXURRADA CORRELACIONADOS COM PERDAS DE SOLO E ERODIBILIDADE DE UM LATOSSOLO ROXO DE DOURADOS (MS) ${ }^{(1)}$
}

\author{
M. P. CARVALHO ${ }^{(2)} \&$ L. C. HERNANI (3)
}

\begin{abstract}
RESUMO
E studaram-se as correlações entre os parâmetros de erosi vidade e as perdas de solo ocorridas de 24/06/1989 a 22/10/1994, num Latossolo Roxo da Unidade de Execução de Pesquisa de Âmbito Estadual (UEPAE) de Dourados (MS) EMBRAPA. Parâmetros de erosividade da chuva, da enxurrada e da chuvaenxurrada foram analisados por meio de regressão linear simples, múltipla e não-linear, com os objetivos de: (a) definir um parâmetro de erosividade para chuvas individuais, visando aprimorar o uso da equação universal de perda de solo local, e (b) determinar o fator erodibilidade para o solo estudado. Foram tomadas 147 chuvas individuais para o cálculo computacional da erosividade. 0 modelo com a altura da enxurrada na forma não-linear a $\left(V_{u}\right)^{b}$ foi o que melhor se correlacionou com a capacidade erosiva das chuvas individuais, superando todos os demais model os, si mples ou compostos, da chuva ou da chuva-enxurrada. Surpreendentemente, neste trabalho, o parâmetro $\mathrm{EI}_{30}$ apresentou baixa correlação com as perdas de solo. Assim, o melhor modelo de erosividade estimador da perda de solo local foi dado pelo seguinte modelo da enxurrada $0,1444\left(V_{u}\right)^{1,0728}$. Os fatores erodibilidade do solo, estimados pelos modelos a $\left(V_{u}\right)^{b} e$ $\mathrm{a}+\mathrm{b} \mathrm{EI}_{30}$, foram, respectivamente, de $0,1444 \mathrm{t} \mathrm{ha}^{-1} \mathrm{~mm}^{-1} \mathrm{e} 0,0037 \mathrm{t}$ ha $\mathrm{h} \mathrm{ha} \mathrm{MJ}^{-1} \mathrm{~mm}^{-1}$.

Termos de indexação: erosão do solo, manejo do solo, modelagem matemática da erosão do solo, equação universal de perda de solo modificada.
\end{abstract}

\footnotetext{
(1) Trabalho apresentado em parte no XXVII Congresso Brasileiro de Ciência do Solo - Brasília (DF ), 1999. Recebido para publicação em novembro de 1999 e aprovado em outubro de 2000.

(2) Professor Assistente do Departamento de Ciência do Solo e Engenharia Rural. Faculdade de Engenharia - FEIS/UNESP. Caixa Postal 31, CEP 15385-000. I lha Solteira (SP). E mail: morel @agr.feis.unesp.br

(3) Pesquisador da EMBRAPA - Centro de Pesquisa Agropecuária do Oeste, Caixa Postal 661, CEP 79804-970. Dourados (MS). Email: hernani@cpao.embrapa.br
} 


\title{
SUMMARY: RAINFALL-RUNOFF EROSIVITY PARAMETERS CORRELATED WITH SOIL LOSSES AND ERODIBILITY OF AN OXISOL FROM DOURADOS, SOUTHERN MATO GROSSO, BRAZIL
}

\begin{abstract}
This study analyses thecorrelations among erosi vity parameters and soil losses, between J une24, 1979 and October 10, 1994, in an Oxisol from theUnidadedeE xecução dePesquisa de Âmbito Estadual at Dourados - EMBRAPA, Southern Mato Grosso, Brazil. Rainfall erosivity, runoff, and rainfall-runoff parameters wereanalysed through simpleand multiple linear regression and nonlinear regression, to: (a) definean erosivity parameter for individual storms, aiming to improve the use of the USLE at that site; (b) obtain the erodibility factor for the soil plot. A set of 147 erosi veindividual storms was analysed and cal culated using a computer program. Therunoff volumeparameter in thenonl inear form was correlated with the erosive capability of individual storms, overcoming all the other si mple or composed, rainfall or rainfall-runoff parameters. Surprisinly, in this paper, theEI ${ }_{30}$ parameter showed a low relationship with the soil losses. Thus, the best erosivity parameter to estimate soil losses at that sitewas the $0.1444\left(\mathrm{~V}_{\mathrm{u}}\right)^{1.0728}$. Theerodi bi lity factors estimated for the parameters $\mathrm{a}(\mathrm{Vu}) \mathrm{b}$ and $\mathrm{a}+\mathrm{b} . \mathrm{EI}_{30}$ were, respectively, $0.1444 \mathrm{tha}^{-1} \mathrm{~mm}^{-1}$ and 0.0037 tha $\mathrm{h} \mathrm{ha} \mathrm{I}^{-1} \mathrm{MJ}^{-1} \mathrm{~mm}^{-1}$.

Index terms: soil erosion, soil management, mathematical modelling of soil erosion, modified universal soil loss equation.
\end{abstract}

\section{NTRODUÇÃO}

A equação universal de perda de solo relaciona os principais fatores que influenciam a erosão acelerada do solo, a saber: erosividade da chuva, erodibilidade do solo, comprimento e grau de inclinação do terreno, cobertura vegetal e manejo da cultura e práticas conservacionistas. Sua utilização tem o objetivo de predizer as perdas médias anuais de solo que poderão ocorrer em determinado local sob uso agrícola (Wischmeier \& Smith, 1965). Erosividade, definida como sendo o potencial da chuva em causar erosão, foi descrita exclusivamente como função das características intrínsecas da própria chuva, dentre as quais o seu volume, intensidade, diâmetro e vel ocidadeterminal da gota e energia cinética.

Desta forma, a pesquisa que era desenvolvida naquela época, centrada na década de 60, vinha demonstrando que as características da chuva que proporcionavam as correlações mais el evadas com a erosão eram a intensidade e a energia cinética (Wischmeier \& Smith, 1958; Hudson, 1973). Em sua forma original, o fator erosividade foi representado pelo parâmetro $\mathrm{EI}_{30}$, dado pelo produto da energia cinética do impacto da gota pela intensi dademáxima em 30 min, sendo, assim, composto exd usivamente de variáveis da chuva. Inicialmente, foi amplamente utilizado nos Estados Unidos (Wischmeier \& Smith, 1965,1978 ) e difundido em todo o mundo.

No Brasil, inúmeras pesquisas constataram a eficácia do parâmetro $\mathrm{EI}_{30}$, dentre as quais as de Lombardi Neto (1977), Morais et al. (1988), Carvalho et al . (1989), Cantalice \& Margol is (1993), Al buquerque et al . (1994), J acob (1995), Silva et al. (1997), Marques et al. (1997).

Paralelamentea tais acontecimentos, Wischmeier (1972) havia sugerido adicionar ao $\mathrm{El}_{30}$ um parâmetro preditor da erosividade da enxurrada. $\mathrm{Na}$ oportunidade, era feita alusão ao fato de que a energia cinética, avaliada pelo fator $E$, estava intimamente associada ao impacto da gota da chuva com o solo, ocorrendo o mesmo entre o fator $\mathrm{I}_{30}$ e o atrito da enxurrada com os sulcos.

Posteriormente, o mesmo pesquisador enfatizou que, no processo de causa e efeito da erosão do solo, respectivamente desempenhado pela chuva e pelo solo, a equação universal de perda desolo considerava, para fins de cálculo, apenas o fator erosividade da chuva como causa. Enfatizou também que sua estimativa da perda média anual de solo era absolutamente satisfatória para isso. Entretanto, quando destinada a projetos em que era premente a necessidade de se conhecerem as perdas de solo de chuvas individuais, tal estimativa diferia tremendamente dos dados observados (Wischmeir, 1976).

Ao final dos anos 60 s e início dos anos 70 s, foi proposta a divisão do processo de erosão do sol o em erosão entressulcos e em sulcos, respectivamente, causadas pela ação da gota da chuva e da enxurrada (Meyer \& Wischmeier, 1969; Young \& Wiersma, 1973; Foster \& Meyer, 1975; Meyer et al., 1975; Mutchler \& Young, 1975). Dessa forma, F oster et al. (1977a,b), estudando os princípios básicos da erosão, concluíram que a estimativa precisa da perda de solo para 
chuvas individuais necessitava da adição de um parâmetro que levasse em conta a erosividade da enxurrada. Na oportunidade, propuseram um parâmetro de erosividade da chuva-enxurrada em substituição ao tradicional $\mathrm{EI}_{30}$, composto pelo somatório das parcelas responsáveis pela erosão entressulcos (chuva) e em sulcos (enxurrada).

Assim, a partir da idealização do novo fator $R$, despontaram algumas pesquisas que visavam à validação deum parâmetro da chuva-enxurrada para chuvas individuais (Lombardi N eto, 1979; Foster et al., 1982). Comotais pesquisas consideravam, como variáveis independentes, as características da chuva, da enxurrada eda chuva-enxurrada, el as ratificaram o melhor desempenho, tanto da erosividade da enxurrada como o da chuva-enxurrada, sobre aquela da chuva. Como exemplo, foi evidenciada a eficácia da erosi vidadeda enxurrada, normalmente até então representada pelo seu volume e pelo pico da taxa de descarga, sobre aquela da chuva, principalmente sobre o parâmetroEI 30 (Dragoun, 1962; Williams et al., 1971; Onstad \& F oster, 1975; Williams, 1975).

Em condições brasileiras, a partir do início dos anos 90s, começaram a aparecer as primeiras pesquisas com a intenção de testar o efeito da erosividade da enxurrada no solo (Carval ho, 1992; Carvalho et al., 1993, 1997), com vistas em adicionar tal componente ao fator $\mathrm{R}$. Tais pesquisas têm demonstrado que, no geral, os fatores erosividade da enxurrada, na forma linear ou na forma potencial, vêm apresentando substancial supremacia, principalmente sobre aqueles da chuva (Carvalho, 1992; Carvalho et al., 1993, 1997; J acob, 1995; Albuquerque, 1997; Albuquerqueet al., 1998).

Dessa forma, os objetivos do presente trabalho, para as condições de Dourados (MS), foram: (a) definir ofator erosividade para chuvas individuais na tentativa de avaliar a eficiência entre si de parâmetros da chuva, da enxurrada e da chuvaenxurrada, visando aprimorar o uso da equação universal de perda de sol o do local, e (b) determinar o fator er odibilidade para o sol o da parcela de campo estudada.

\section{MATE RIAL E MÉTODOS}

\section{Plano experimental}

Este trabalho utilizou dados pluviográficos e de perda de solo e enxurrada, col etados na Unidade de Execução de Pesquisa de Âmbito Estadual (UEPAE) de Dourados (MS), EMBRAPA, Ministério da Agricultura, Brasil, localizada na longitude 54\%49'W e na latitude 22014 'S, com uma altitude média de 452 m, em área total de 400 ha. As médias anuais depreci pitação ede temperatura são respectivamente de $1.350 \mathrm{~mm}$ e $22^{\circ} \mathrm{C}$. O tipo climático é $A w$, segundo Koeppen, definido como tropical úmido com estação chuvosa no verão e seca no inverno. O solo onde a parcela de campo ficou instalada é um Latossolo Roxo distrófico/epieutrófico muito argiloso (Brasil, 1989) (Latossolo Vermelho Aluminoférrico típico muito argiloso(4)).

Os dados de perda de solo e de enxurrada originaram-se de uma parcela de campo com $0,03 \mathrm{~m} \mathrm{~m}^{-1}$ de declive, $77,0 \mathrm{~m}^{2}$ de área $(22,0 \mathrm{~m}$ de comprimento no senti do do decl ive), conectada a um coletor do tipo Gei b para fração de 1/7 (Bertoni, 1949; Marques, 1951). O manejo do sol o foi o mesmo que o da parcela-padrão, isto é, em alqueive contínuo (Wischmeier, 1972). Assim, os dados de perda de enxurrada, por serem uma variável independente, não sofreram nenhuma correção, enquanto os dados de perda de sol o foram corrigidos para as condições de parcela-padrão, por meio da seguinte equação de Bertoni (1959):

$$
A_{J B}=0,018 S^{1,18} L^{1,63}
$$

O declive e o comprimento da parcela de campo utilizados foram, respectivamente, de $0,03 \mathrm{~m} \mathrm{~m}^{-1}$ (3\%) ede $22 \mathrm{~m}$, ao passo que, nas condições de parcelapadrão, foram, respectivamente, de 0,09 $\mathrm{m} \mathrm{m}^{-1}$ (9\%) e de 22,13 m. A substitui ção desses dados na equação (1) resultou nas quantias de perda de solo $\left(A_{J}\right)$ de 10,1490 e 37,4626 kg/unidade delargura, respectivamente, para as condições de parcelas de campo e padrão. Assim, a relação 37,4626/10,1490 proporcionou o fator de conversão igual a 3,6913, necessário para a transformação das perdas de solo da condição de parcela de campo para parcela-padrão. A equação final, utilizada para a correção de tais dados, foi a seguinte:

$$
A_{\text {cor }}=3,6913 A_{\text {cam }}
$$

O pluviógrafo utilizado, tipo sifão IH e modelo Hillman-4, registrou incrementos sucessivos de chuva, assim como o total acumulado. Apresentou autonomia para registrar chuvas num intervalo de $24 \mathrm{~h}$, sendo movido a corda por meio de um mecanismo derelógio. As chuvas individuais erosivas estudadas (Wischmeier, 1959; Wischmeier \& Smith, 1978; Cabeda(5)) e selecionadas de acordo com os mesmos critérios que os de Carvalho et al. (1993; 1997) foram obti das em pluviogramas CED-RE-843, os quais registraram a quantidade de chuva e o tempo de ocorrência, respectivamente, na ordenada e na abscissa. Sua amplitude de registro variou de $-1,0$ e 11,0 mm de precipitação, a intervalos de $0,2 \mathrm{~mm}$. O registro de tempo foi efetuado durante $24 \mathrm{~h}$, a intervalos de $10 \mathrm{~min}$.

\footnotetext{
(4) HERNANI, L.C. Comunicação pessoal. 1999. (Centro de Pesquisa Agropecuária do Oeste, EMBRAPA, Dourados, Mato Grosso do Sul, Brasil).

(5) CABEDA, M.S.V. Computation of storm Elvalues. West Lafayette, Purdue University, 1976. 6p. (unpublished).
} 
Analisaram-se dados pluviográficos, de perda de solo e de enxurrada, acumulados entre 24/06/1989e $22 / 10 / 1994$. F oram separadas 116 chuvas individuais erosivas, assim como 31 não-erosivas, mas que proporcionaram significativas perdas de solo e, ou, enxurrada. Os pontos de inflexão da intensidade das 147 chuvas foram cotados e extraídos visual mente, uma vez que o formato convexo da ordenada (altura pluviométrica) impossibilitou o uso da mesa digitalizadora para a extração dos pares cartesianos. Em seguida, efetuou-se o processamento dos dados, através de um programa computacional para cálculo da erosividade da chuva, no Departamento de Ciência do Solo e Engenharia Rural da Faculdade deEngenharia -UNESP/Campus dellha Solteira (SP). O referido programa foi processado numa calculadora manual Casio FX-880P, em linguagem Basic.

Para compor os model os matemáticos representativos dos parâmetros de erosividade da chuva, da enxurrada e da chuva-enxurrada, foram extraídas, de cada chuva, as seguintes variáveis independentes: (a) altura total da chuva, $\mathrm{V}_{\mathrm{r}}(\mathrm{mm}) ;(\mathrm{b}) \mathrm{I}_{5} \mathrm{el}_{30}$, sendo, respectivamente, as intensidades máximas das chuvas em 5 e em 30 min $\left(\mathrm{mm} \mathrm{h}^{-1}\right)$, de acordo com a seguinte expressão, apresentada por Carval ho et al . (1989):

$$
\mathrm{I}_{\mathrm{n}}=\left(\sum_{\mathrm{i}=1}^{\mu} \mathrm{i}_{\mu} \cdot \mathrm{t}_{\mu}\right) / \mathrm{n}
$$

(c) $\mathrm{EI}_{30}$, sendo o parâmetro de erosividade da chuva proposto por Wischmeier (1959), dado pelo produto da sua energia cinética total pela intensidade máxima em $30 \min (M J) \mathrm{mm} \mathrm{ha}^{-1} \mathrm{~h}^{-1}$ ).

Para calcular a referida energia, foi utilizada a seguinte equação (F oster et al., 1981):

$$
E=0,119+0,0873 \log I
$$

(d) altura total da enxurrada, $\mathrm{Vu}(\mathrm{mm})$, obtida diretamente no tanque col etor da parcel a de campo; (e) EI A, sendo o parâmetro de erosividade da chuva durantea ocorrência da enxurrada, apresentado por Lombardi Neto (1979) eF oster et al . (1982) $\left(\mathrm{mm}^{2} \mathrm{~h}^{-1}\right)$, $\mathrm{e}(\mathrm{f}) \alpha=\left(\mathrm{V}_{\mathrm{u}} N_{\mathrm{r}}\right)^{1 / 2}$, coeficiente adimensional de proporcionalidade entrea enxurrada ea chuva apresentado por Lombardi Neto (1979) e F oster et al. (1982).

\section{Seleção dos parâmetros de erosividade}

A sel eção dos parâmetros de erosividade estudados foi idêntica àquela apresentada por Carvalho et al. (1997), conforme idealização prévia deL ombardi Neto (1979) eF oster et al. (1982). Assim, foram considerados três grupos de parâmetros de erosividade: (a) da chuva; (b) da enxurrada e (c) da chuva-enxurrada.

\section{Parâmetros de erosividade da chuva}

Os parâmetros mais simples de erosividade da chuva, respectivamenterelacionados por Wischmeier
\& Smith (1958) e Wischmeier \& Smith (1965), adotados na forma linear, foram os seguintes:

$$
\begin{aligned}
& \gamma=a+b V_{r} \\
& \gamma=a+b E_{30} .
\end{aligned}
$$

Uma vez que Wischmeier \& Smith (1978) haviam relatado que o cál cul o do $\mathrm{EI}_{30}$ é mor oso e complicado, foram adotados os seguintes parâmetros, conforme sugestões de F oster \& Meyer (1975):

$$
\begin{gathered}
\mathrm{EI}_{30} \sim \mathrm{a}+\mathrm{b}\left(\mathrm{V}_{\mathrm{r}}\right) \mathrm{I}_{30} \\
\mathrm{EI}_{30} \sim \mathrm{a}+\mathrm{b}\left(\mathrm{V}_{\mathrm{r}}\right) \mathrm{I}_{30} 1,14 \\
\mathrm{EI}_{30} \sim \mathrm{a}+\mathrm{b}\left(0,119+0,0873 \operatorname{logl}_{30}\right) \mathrm{V}_{\mathrm{r}} \mathrm{I}_{30} \\
\mathrm{EI}_{30} \sim \mathrm{a}+\mathrm{b}\left(\mathrm{V}_{\mathrm{r}}\right)^{\mathrm{b}}
\end{gathered}
$$

O parâmetro de erosividade da chuva mais complexo e pesquisado pel o presente estudo foi oEIA (Lombardi Neto, 1979; Foster et al.,1982). Tal complexidade ocorre pela presença na sua composição de variáveis de maior dificuldade de obtenção, tais como o $\mathrm{V}_{\mathrm{u}}$ e o $\mathrm{I}_{30}$. Dessa forma, este parâmetro avalia a erosividade da chuva apenas durante a ocorrência da enxurrada. Sua expressão, cuja determinação encontra-se detal hadamente explicada em Carval ho et al. (1997), éa seguinte:

$$
E I A=I_{30}\left(V_{u} \cdot V_{r}\right)^{1 / 2}
$$

\section{Parâmetros de erosividade da enxurrada}

O parâmetro mais simples da enxurrada que pode expressar sua erosividade depende linearmente da altura da enxurrada $\left(\mathrm{V}_{\mathrm{u}}\right)$, conforme a seguinte expressão (Carval ho et al., 1997):

$$
\gamma=\mathrm{a}+\mathrm{bV}_{\mathrm{u}}
$$

Contudo, como Willians (1975) havia observado que a combinação desse vol ume com o pico da taxa da enxurrada $\left(\sigma_{\mathrm{pu}}\right)$, na forma potencial, apresentava melhores resultados, foram propostos os seguintes parâmetros:

$$
\begin{aligned}
& R_{M}=a\left(V_{u} \alpha I_{30}\right)^{b} \\
& R_{L}=a\left(V_{u} \alpha I_{5}\right)^{b}
\end{aligned}
$$

\section{Parâmetros de erosividadeda chuva-enxurrada}

Considerando as inúmeras evidências das observações teóricas, assim como os dados de pesquisa de campo, foi sugerido que o fator erosividade da chuva, até então exclusivamente representado pelo $\mathrm{EI}_{30}$, deveria conter termos distintos para as formas de erosão nos entressulcos e nos sulcos, respectivamente, desencadeadas pelo impacto da gota da chuva e pel o atrito da enxurrada. A representação matemática desse conceito, estabelecida por Foster et al. (1977a,b), foi a seguinte: 


$$
\mathrm{R}_{\mathrm{F}}=\mathrm{R}_{\mathrm{r}}+\mathrm{R}_{\mathrm{u}}
$$

Assim, no presente estudo, representando a erosão nos entressulcos $\left(R_{r}\right)$, pel os parâmetros $\mathrm{EI}_{30}$ e EIA, assim como a erosão em sulcos $\left(R_{u}\right)$, pelo volume da enxurrada $\left(V_{u}\right)$, por meio das combinações possíveis, obtiveram-se os parâmetros de erosividade da chuva-enxurrada mais simples, representados basicamente pel o seguinte model o geral:

$$
R_{\mathrm{F}}=\mathrm{a}+\mathrm{b} \mathrm{R}_{\mathrm{r}}+\mathrm{c} \mathrm{R}_{\mathrm{u}} .
$$

Valendo do conceito estabelecido pela equação (15), assim como da utilização dos parâmetros $\mathrm{EI}_{30}$ e EIA para representação do seu termo $R_{r}$, foram pesquisadas as seguintes expressões, para representação dotermo compl ementar de erosividade da enxurrada $\left(R_{u}\right)$, relacionadas a seguir pelas equações (17), (18) e (19). Portanto, aquel a de Onstad \& F oster (1975):

$$
\mathrm{R}_{\mathrm{o}}=\mathrm{a}\left(\mathrm{V}_{\mathrm{u}}\right) \sigma_{\mathrm{pu}}^{1 / 3}
$$

Também, o termo de Foster e Meyer (1975) e Foster et al. (1977a):

$$
R_{F M}=\alpha S\left(\tau_{E}-\tau_{C}\right) \xi
$$

E, finalmente, aquela idealizada por Foster \& Meyer (1972) e proposta por Lombardi Neto (1979) e F oster et al. (1982):

$$
R_{\mathrm{LF}}=\mathrm{a}(\mathrm{Vu}) \exp \left(\mathrm{d} / \alpha . \mathrm{I}_{5} \text { e ou } \mathrm{I}_{30}\right)
$$

\section{Não-linearidade dos parâmetros de erosividade}

Alguns parâmetros de erosividade da chuva eda enxurrada foram propostos nas formas linear e nãolinear, com vistas em pesquisar a existência de correl ação de forma não-linear entre eles. Esse fato foi inicialmente proposto para as condições norteamericanas (Williams, 1975; F oster et al., 1982), e, posteriormente, para as condições brasileiras (Lombardi Neto, 1979; J acobs, 1995; Albuquerque, 1997; Carvalho et al., 1997; Marques et al., 1997), uma vez que a linearidade existente entre a perda desolo e o $\mathrm{EI}_{30}$ simplifica a consi deração da interação de uma distribuição não-uniforme do $\mathrm{EI}_{30}$ anual ea distribuição não-uniforme da razão de perda de solo da equação universal de perda de solo (F oster et al., 1982).

\section{Análise estatística}

Do total das 147 chuvas estudadas, algumas apresentavam valor nulo de perda de solo. Assim, atribuiu-se o valor de $10^{-7} \mathrm{t} \mathrm{ha}^{-1}$ para tais perdas, para que o estudo de regressão pudesse ser efetuado. Por essa razão, foram utilizadas regressões lineares simples e múltiplas, para os parâmetros lineares, e regressões não-lineares, para os parâmetros nãolineares, a fim de ajustar os 24 parâmetros de erosividade estudados (Quadro 1). As análises nãolineares foram efetuadas pelo método de GaussNewton. Empregou-se o coeficiente de correlação para os model os lineares simples, assim como para aqueles lineares por anamorfose, visando verificar a homogeneidade entre tais coeficientes, conforme Graybill (1976). Apresentaram-se os coeficientes de regressão (a, b, ce d) dos parâmetros de erosividade ajustados e, finalmente, aplicou-se o teste $t$ de Student, para observar a significância estatística dos coeficientes $\mathrm{R}^{2}$ ajustados, segundo Hoffmann \& Vieira (1977).

\section{RESULTADOS E DISCUSSÃO}

Observou-se diferença significativa entre os coeficientes de correlação ( $r$ ) do parâmetro nãolinear, representados pela equação \#1, e aqueles representados pelas equações \#2, \#3 e \#4 (Quadro 1). Portanto, o parâmetro não-linear $y=a\left(V_{u}\right)^{b}$, por ser de fácil obtenção e de el evada correlação com a erosão, deve ser perfeitamente indicado para a estimativa das perdas desolo causadas pelas chuvas individuais de Dourados. Esse fato demonstrou a supremacia desse parâmetro sobre aqueles do $\mathrm{EI}_{30}$ de Wischmeier \& Smith (1958), (\#17 e, ou, \#20), quando o objetivo era estimar a erosividade das chuvas individuais para olocal. Por outrolado, esse resultado atestou as idéias de Dragoun (1962), Williams et al. (1971), Onstad \& Foster (1975), Williams (1975), Foster et al. (1977a,b), Lombardi Neto (1979), F oster et al. (1982), Carvalho (1992), Carvalho et al. (1993, 1997), J acobs (1995), Albuquerque (1997) e Al buquerqueet al. (1998), que preconizaram, para a equação universal de perda de solo, um fator erosividade da enxurrada.

\section{Parâmetros de erosividade da chuva}

A análise dos dez parâmetros de erosividade da chuva estudados indicou que o EIA, na forma nãolinear (\#2, Quadro 1), apresentou-se como o mel hor entre eles, superando, inclusive, todos aqueles da chuva-enxurrada. Contudo, deve-se considerar que oEIA não é um autêntico parâmetro de erosividade da chuva, uma vez que contém em sua fórmula a altura da enxurrada $\left(\mathrm{V}_{\mathrm{u}}\right)$, conforme pode ser observado na equação (11). Por outro lado, os parâmetros de erosividade restantes (\#16 ao \#24, Quadro 1) foram os de mais baixa correlação com as perdas de solo, concordando este fato com os relatos de Lombardi Neto (1979), F oster et al. (1982), J acobs (1995), Carval ho et al. (1997) e Al buquerque(1997).

Dentre os parâmetros de erosividade da chuva compreendidos entre o \#16 e o \#24, o model o linear múltipl o que contém o $\mathrm{EI}_{30}$ eo EI A (\#16) apresentouse superior aos demais (Quadro 1 ). Também, a igualdade estatística verificada nos parâmetros estabel ecidos entre o \#18 e o \#23 indicou que, para 
Quadro 1. Equações de regressão, coeficientes de determinação $\left(r^{2}\right)$, de correlação $(r)$ e de regressão (a, b, c e d) ajustados entre as perdas de solo em condições de alqueive contínuo e parâmetros de erosividade da chuva e da enaxurrada, para 147 chuvas ocorridas em Dourados (MS) entre 24/06/1989 e 22/10/1994

\begin{tabular}{|c|c|c|c|c|c|c|c|c|}
\hline \multicolumn{3}{|c|}{ Equação } & \multirow{2}{*}{$\mathbf{R}^{2}(\mathbf{2})$} & \multirow{2}{*}{$r^{(3)}$} & \multicolumn{4}{|c|}{ Coeficiente de regressão } \\
\hline № & Modelo matemático & Tipo(1) & & & $\mathbf{a}$ & b & c & d \\
\hline$\# 1$ & $\gamma=\mathrm{a}\left(\mathrm{V}_{\mathrm{u}}\right)^{\mathrm{b}}$ & en & 0,9944 & 0,9972a & $1,4438.10^{-1}$ & 1,0728 & -- & -- \\
\hline$\# 2$ & $\gamma=a(E I A)^{b}$ & ch & 0,9145 & $0,9563 b$ & $1,1739.10^{-2}$ & $1,8934 \cdot 10^{-1}$ & -- & -- \\
\hline \#3 & $\gamma=a\left(V_{u} \cdot \alpha \cdot I_{5}\right)^{b}$ & en & 0,8976 & $0,9474 b$ & $1,1394.10^{-2}$ & $1,8234 \cdot 10^{-1}$ & -- & -- \\
\hline \#4 & $\gamma=a\left(V_{u} \cdot \alpha \cdot I_{30}\right)^{b}$ & en & 0,8825 & $0,9394 b$ & $1,1191.10^{-2}$ & $1,7312.10^{-1}$ & -- & -- \\
\hline \#5 & $\gamma=a E I_{30}+b\left(V_{u}\right) \exp .\left(c / \alpha . I_{5}\right)$ & ch/en & 0,8604 & -- & $2,8243.10^{-3}$ & $1,6043 \cdot 10^{-1}$ & $1,2752 \cdot 10^{-1}$ & -- \\
\hline \#6 & $\gamma=a \operatorname{EIA}+b\left(V_{u}\right) \exp .\left(c / \alpha . I_{5}\right)$ & ch/en & 0,8380 & -- & $1,2650.10^{-3}$ & $1,3634.10^{-1}$ & $1,3549.10^{-1}$ & -- \\
\hline$\# 7$ & $\gamma=a\left(E I_{30}\right)^{b}+c\left(V_{u}\right) \exp .\left(d / \alpha . I_{5}\right)$ & ch/en & 0,8232 & -- & $-1,0896.10^{-2}$ & $-1,1121$ & $1,9501 \cdot 10^{-1}$ & $1,2760 \cdot 10^{-1}$ \\
\hline \#8 & $\gamma=a(E \mid A)^{b}+c\left(V_{u}\right) \exp .\left(d / \alpha . I_{5}\right)$ & ch/en & 0,8111 & -- & $-7,2465 \cdot 10^{-3}$ & $-9,5583 \cdot 10^{-1}$ & $1,9489.10^{-1}$ & $1,3321 \cdot 10^{-1}$ \\
\hline \#9 & $\gamma=a+b E I_{30}+c \cdot V_{u}$ & ch/en & 0,6921 & -- & $1,4115.10^{-1}$ & $2,5310 \cdot 10^{-3}$ & $1,5998.10^{-1}$ & -- \\
\hline$\# 10$ & $\gamma=a+b E I A+c . V_{u}$ & ch/en & 0,6624 & -- & $2,5780.10^{-1}$ & $1,2720 \cdot 10^{-3}$ & $1,2796.10^{-1}$ & -- \\
\hline$\# 11$ & $\gamma=a+b V_{u}$ & en & 0,5800 & $0,7616 \mathrm{c}$ & $2,6422 \cdot 10^{-1}$ & $1,2001 \cdot 10^{-1}$ & -- & -- \\
\hline$\# 12$ & $\gamma=a+b E I A+c . V_{u}\left(\alpha \cdot I_{5}\right)^{1 / 3}$ & ch/en & 0,5699 & -- & $3,9985.10^{-1}$ & $2,0760 \cdot 10^{-3}$ & $1,7268.10^{-2}$ & -- \\
\hline$\# 13$ & $\gamma=a+b E I A+c . V_{u}\left(\alpha \cdot I_{30}\right)^{1 / 3}$ & ch/en & 0,5646 & -- & $4,1664 \cdot 10^{-1}$ & $3,1850 \cdot 10^{-3}$ & $2,0044 \cdot 10^{-2}$ & -- \\
\hline$\# 14$ & $\gamma=a+b(1 / 2) E I_{30}+c(1 / 2) V_{u}\left(\alpha . I_{5}\right)^{1 / 3}$ & ch/en & 0,5600 & -- & $3,1827.10^{-1}$ & $6,3200 \cdot 10^{-3}$ & $6,1329 \cdot 10^{-2}$ & -- \\
\hline \#15 & $\gamma=a+b(1 / 2) E I_{30}+c(1 / 2) V_{u}\left(\alpha . I_{30}\right)^{1 / 3}$ & ch/en & 0,5469 & -- & $3,4601 \cdot 10^{-1}$ & $6,3970 \cdot 10^{-3}$ & $7,8148.10^{-2}$ & -- \\
\hline \#16 & $\gamma=a+b E_{30}+c . E I A$ & $\mathrm{ch}$ & 0,5383 & -- & $6,1736 \cdot 10^{-1}$ & $-4,8734 \cdot 10^{-4}$ & $4,1634 \cdot 10^{-3}$ & -- \\
\hline \#17 & $\gamma=a+b E I_{30}$ & ch & 0,5373 & $0,7330 \mathrm{c}$ & $1,6548.10^{-1}$ & $3,7230 \cdot 10^{-3}$ & -- & -- \\
\hline \#18 & $\gamma=a+b V_{r}$ & ch & 0,2970 & $0,5450 \mathrm{~d}$ & $-4,8302 \cdot 10^{-1}$ & $5,2394 \cdot 10^{-2}$ & -- & -- \\
\hline \#19 & $\gamma=a+b E I A$ & ch & 0,2336 & 0,4833 de & $3,4938 \cdot 10^{-1}$ & $9,3600 \cdot 10^{-4}$ & -- & -- \\
\hline$\# 20$ & $\gamma=\mathrm{a}\left(\mathrm{E} I_{30}\right)^{\mathrm{b}}$ & ch & 0,1790 & $0,4231 \mathrm{de}$ & $6,7501 \cdot 10^{-10}$ & 3,3931 & -- & -- \\
\hline$\# 21$ & $\gamma=\mathrm{a}\left(\mathrm{V}_{\mathrm{r}}\right)^{\mathrm{b}}$ & ch & 0,1480 & 0,3847 de & $2,9379 \cdot 10^{-11}$ & 6,0721 & -- & -- \\
\hline$\# 22$ & $\gamma=a+b\left(V_{r}\right) I_{30}$ & ch & 0,1471 & 0,3835 de & $2,9344.10^{-1}$ & $8,3900 \cdot 10^{-4}$ & -- & -- \\
\hline \#23 & $\gamma=a+\left.b\left(V_{r}\right)\right|_{30} 1,14$ & ch & 0,1401 & $0,3743 \mathrm{de}$ & $2,8397.10^{-1}$ & $7,4901 \cdot 10^{-4}$ & -- & -- \\
\hline$\# 24$ & $\gamma=a+b\left(0,119+0,\left.0873 \log \right|_{30}\right) V_{r} . I_{30}$ & ch & 0,1246 & $0,3530 \mathrm{e}$ & $1,6335.10^{-1}$ & $7,8407 \cdot 10^{-4}$ & -- & -- \\
\hline
\end{tabular}

(1) Tipo do parâmetro de erosividade: ch =chuva, en =enxurrada, ch/en =chuva-enxurrada. (2) Todos os coeficientes de determinação foram significativos a $1 \%$ pelo teste $t .{ }^{(3)}$ Coeficientes de correlação seguidos pela mesma letra não diferem entre si a $5 \%$ pelo teste de Graybill.

as condições de Dourados, a tentativa desimplificação do cál culo do $\mathrm{EI}_{30}$, por meio do uso do $\mathrm{V}_{\mathrm{r}} \mathrm{e}, \mathrm{ou}$, do $\mathrm{I}_{30}$, conforme preconizado por Foster \& Meyer (1975), não poderia ser recomendada pelo fato de o tradicional parâmetro $\mathrm{EI}_{30}$ (\#17) de Wischmeier \& Smith (1965) apresentar-se estatisticamente superior aos referidos parâmetros (\#18 ao \#23), discordando do observado por Lombardi Neto (1979), F oster et al. (1982) e Carvalho et al . (1997).

\section{Parâmetros de erosividade da enxurrada}

Os parâmetros não-lineares de erosividade da enxurrada (\#1, \#3 e \#4, Quadro 1), por apresentarem as mais el evadas correlações com a erosão, foram os melhores do presente estudo. Detalhando-se a análise, o estudo estatístico aplicado revel ou que o parâmetro não-linear de erosividade da enxurrada $\gamma=\mathrm{a}\left(\mathrm{V}_{\mathrm{u}}\right)^{\mathrm{b}}(\# 1)$ comportou-se como o melhor entre todos. Dessa forma, esse fato vem a revolucionar a pesquisa do fator erosividade da chuva $(R)$, da equação universal de perda de sol o, para as condições de Dourados. O parâmetro $\gamma=a\left(V_{u}\right)^{b}(\# 1)$, quando analisado pelo coeficiente de determinação $\left(R^{2}\right)$, apresentou uma correlação com a perda de solo da ordem de $45,71 \%$ superior àquela do parâmetro $\gamma=$ $\mathrm{a}+\mathrm{b} . \mathrm{EI}_{30}$ (\#17). Também, no confronto direto entre $\mathrm{oV}_{\mathrm{u}} \mathrm{eoEI}_{30}$, duas variáveis independentes, a primeira mostrou-se sobremaneira mais vantajosa, devido, entre outras, à sua substancial facilidadedeobtenção

Assim, considerando as condições do presente estudo, pôde-se concluir que o fator $\mathrm{R}$ local deve ser determinado por meio do parâmetro $\gamma=a\left(V_{u}\right)^{b}$, em detrimento do de Wischmeier (1959), representado pelo $\mathrm{EI}_{30}$ (\#17). Para as condições brasileiras, tal fato já havia sido apresentado por Carvalho (1992), Carvalho et al. (1997) e Albuquerque (1997).

O modelo linear simples com a altura da enxurrada $V_{u}$ (\#11, Quadro 1) foi estatisticamente igual ao modelo linear simples com o $\mathrm{EI}_{30}$ (\#17). No entanto, foi superior aos model os representados pelo $\mathrm{V}_{\mathrm{r}}$ (\#18) e o EIA (\#19). Portanto, para as condições brasileiras, em relação à comparação entre o $V_{\mathrm{u}}$ e o 
$\mathrm{EI}_{30}$, este fato concordou com Albuquerque (1997) e discordou de Carval ho (1992) e Carvalhoet al. (1997). $\mathrm{J}$ á com relação à comparação entre o $\mathrm{V}_{\mathrm{u}}$ e o $\mathrm{V}_{\mathrm{r}}$, este fato concordou com Carvalho(1992), Carval ho et al . (1997) e Albuquerque (1997). Finalmente, com relação à comparação entre o $\mathrm{V}_{u}$ e o $\mathrm{EI} A$, este fato discordou de Carvalho (1992), Carvalho et al. (1997) e Albuquerque (1997).

A supremacia dos parâmetros não-lineares (\#1, \#3 e \#4) sobre o único parâmetro linear (\#11) veio a enfatizar a elevada resposta da erosão local aos parâmetros de erosividade da enxurrada, estabelecidos na forma potencial e na presença ou não do pico da taxa da enxurrada $\left(\sigma_{\mathrm{pu}}\right)$, conforme sugestão de Williams (1975). Este fato ficou plenamente de acordo com o observado para Campinas, Mococa ePindorama, por Carvalho (1992) e Carvalho et al . (1997), assim como com o observado para Sumé (Albuquerque, 1997). Por outro lado, a igualdade existente entre os parâmetros nãolineares \#3 e \#4 evidenciou não haver diferença entre o uso do $I_{5}$ ou do $I_{30}$, como termos adjuntos de a, para a estimativa do pico da taxa da enxurrada, concordando com o observado por Lombardi Neto (1979), Foster et al. (1982), Carval ho (1992), Carval ho et al. (1997) e Albuquerque (1997).

\section{Parâmetros deerosividade da chuva-enxurrada}

Os model os não-lineares (\#5, \#6, \#7, \#8) apresentaram substancial superioridade sobre os lineares múltiplos (\#9, \#10, \#12, \#13,\#14 e \#15) (Quadro 1). Sabendo que os coeficientes de determinação dos model os não-lineares (\#5 ao \#8) variaram entre 0,8604 e 0,8111, sendo, portanto, bastante representativos, tal fato ratificou a eficiência do fator $R$, quando composto dos termos distintos para a erosão entressulcos e em sulcos, conforme estabelecido pela equação (15) de Foster et al. $(1977 a, b)$. Ratificou também o conceito de tensão crítica decisalhamento do solo $\left(\tau_{c}\right)$, estimada pelo termo exp. (d/al $\left.{ }_{5}\right)$, para a erosão em sulcos ocorrida em Dourados, conforme proposições de Foster \& Meyer (1975) e F oster et al. (1977a,b).

Entretanto, evidencia-se quetodos os parâmetros de erosividade da chuva-enxurrada apresentaram desempenho inferior em relação àqueles da enxurrada, estabel ecidos na forma não-linear (\#1, \#3 e \#4), fato esse observado também por Lombardi Neto (1979), Foster et al. (1982), Carvalho (1992) e Carvalho et al. (1997). Portanto, os referidos model os não-lineares foram tidos como impraticáveis, uma vez que o ajuste dos seus coeficientes $c$ e d foi de extrema dificuldade.

Os model os lineares múltiplos mais simples (\#9 e \#10) tiveram mel hor desempenho do que aqueles mais complexos (\#12, \#13, \#14 e \#15), que foram idealizados por Onstad \& Foster (1975), no intuito de se pesquisar a adição do efeito não-linear $\left(\sigma_{\mathrm{pu}}\right)^{1 / 3}$ à taxa da enxurrada. Também, nesse caso, pareceu não haver diferença entre o uso do $\mathrm{I}_{5}$ ou do $\mathrm{I}_{30}$ para a estimativa do pico da taxa da enxurrada, que está contida nos parâmetros \#12, \#13, \#14 e \#15 (Quadro 1). Todos esses fatos ficaram plenamente de acordo com o observado por Lombardi N eto (1979), Foster et al. (1982), Carval ho (1992), Carval ho et al. (1997) e Albuquerque (1997).

\section{Não-linearidade dos parâmetros de erosividade}

No presente estudo, o $\mathrm{V}_{\mathrm{r}}$, o $\mathrm{EI}_{30}$ e o $\mathrm{EI} A$ foram estabel ecidos como parâmetros da chuva, assim como o $V_{u}$, como da enxurrada. Entretanto, sabe-se que o EIA, conceituado como um parâmetro de erosividade da chuva durante a ocorrência da enxurrada, possui o termo $V_{u}$, conforme a equação (11). Dessa forma, pôde ser constatado que os model os não-lineares de erosividade da enxurrada (\#1, \#2, \#3 e \#4, Quadro 1), que tiveram um coeficiente de correlação $(r)$ entre 0,9972 e 0,9394, apresentaram um desempenho significativamente superior na explicação da erosão do solo, em relação às respectivas formas lineares, que tiveram um $r$ entre 0,7616 e 0,4833 (\#11 e \#19).

Esse fato concordou plenamente com o obtido por Carvalho (1992) e Carvalho et al. (1997), para as condições do estado de São Paulo, e com o obtido por Albuquerque (1997), para o estado da Paraíba. No entanto, concordou parcialmente com o obtido por Lombardi Neto (1979) e Foster et al. (1982), que verificaram apenas pequena melhoria dessa estimativa para a mesma situação, para as condi ções norte-americanas. Portanto, para Dourados concluise haver el evadíssi ma resposta das perdas desol o por erosão, deforma não-linear, à erosividadeda enxurrada, em vista, provavel mente, das condições reinantes das el evadas intensidades das chuvas tropicais.

Por outro lado, os parâmetros de erosividade da chuva não proporcionaram esse mesmo desempenho. Neste caso, apenas o parâmetro linear $\mathrm{EI}_{30}$ (\#17) superou os demais, estabel ecidos na forma linear e, ou, não-linear (\#18, \#20 e \#21), os quais, estatisticamente, foram iguais entre si. Tal fato concordou com os dados de Carvalho (1992), Carvalho et al. (1997) eAlbuquerque (1997). Concluiu-se, portanto, não haver o mesmo desempenho dos parâmetros não-lineares de erosividade da chuva sobre os seus correspondentes na forma linear, a exemplo do caso dos parâmetros de erosividade da enxurrada.

Considerando as condições deste trabalho, verificou-se el evada correl ação entre os parâmetros não-lineares de erosi vidade da enxurrada eas perdas de solo por erosão. Observando os parâmetros de erosividade da chuva, além de tal correlação nãoter sidoel evada - uma vez que o coeficiente de correlação (r), variando de 0,7330 (\#17) a 0,3847 (\#21), foi de médioa baixo- mostraram-seiguais estatisticamente. É provável quenão só haja uma boa resposta da erosão local aos parâmetros não-lineares de erosividade da 
enxurrada, assim como uma resposta deficitária, tanto dos parâmetros não-lineares quanto dos lineares, representantes da erosividade da chuva, mas também quea excelente afinidade da erosão aos parâmetros não-lineares da enxurrada seja devida às altas intensidades das chuvas convectivas tropicais.

Desta forma, o ajuste do model o potencial com o volume da enxurrada $\left(\mathrm{V}_{\mathrm{u}}\right)(\# 1, \mathrm{Quadro} 1)$ seria realmente o melhor, uma vez que os desvios observados entre os dados e sua curva de regressão seriam os menores. Portanto, a utilização de um parâmetro linear, tal como o $a+b \mathrm{EI}_{30}$ em substituiçãoao referido $a\left(V_{u}\right)^{b}$, para obtenção dofator $\mathrm{R}$ de Dourados, poderia causar uma superestimativa da erosão para chuvas de baixa intensidade, além de causar uma subestimativa para aquelas de el evada intensidade.

Williams (1975) estudou a predição da perda de sol o das chuvas individuais para os Estados Unidos, substituindo o tradicional índice $\mathrm{EI}_{30}$ do fator $\mathrm{R}$ por um parâmetro de erosividade da enxurrada, conforme estabelecido pela equação (14), dada por $a\left(V_{u} \cdot \alpha \cdot I_{5}\right)^{b}$. Concluiu-se que o modelo em questão proporcionou uma estimativa da erosão muito melhor do que aquele tradicional da equação universal de perda de solo. O coeficiente b, ajustado para esse caso, foi igual a 0,56.

Para Dourados, o coeficiente b dos parâmetros não-lineares de erosividade da enxurrada (\#1, \#2, \#3, \#4) variou entre 0,1731 (\#4) e 1,0728 (\#1). Entretanto, nãofoi observadonenhum valor próximo daquele de Williams (1975), que foi de 0,56. Exclusivamente em relação ao modelo $\mathrm{a}\left(\mathrm{V}_{\mathrm{u}}\right)^{\mathrm{b}}(\# 1$, Quadro 1), seu coeficiente b $(1,0728)$ representou quase que o dobro daquele valor observado por Williams (1975). Entretanto, foi semel hanteàqueles observados por Carvalho (1992), para Campinas e Mococa, respectivamente, de 1,3753 e 1,1706, assim como inferior aos observados para Pindorama (Carval ho et al., 1997) eSumé(Albuquerque, 1997), respectivamente, de 0,7783 e 0,8825.

O comportamento de uma função potência, a exemplo do parâmetro $\gamma=\mathrm{a}\left(\mathrm{V}_{\mathrm{u}}\right)^{\mathrm{b}}$ (\#1, Quadro 1$)$, é determinado principalmente pelo seu expoente $b$. Para o caso deser o expoenteb positivo, quanto mai or for o seu valor, mais rápida a resposta ao valor crescente da erosão. Portanto, esse fato vem a enfatizar a diferença existente entre o primeiro parâmetro (\#1) e os três seguintes (\#2, \#3 e \#4), uma vez que o coeficienteb do primeiro $(1,0728)$ foi bastante superior ao dos demais, que foram, respectivamente, de 0,1893; 0,1834 e 0,1731. Sabe-seque o parâmetro \#l écompostoapenas do $V_{u}$, assim como os parâmetros $\# 2$, \#3 e \#4 são compostos das variáveis $V_{u}, V_{r}, I_{5} \mathrm{el}_{30}$. Dessa forma, a existência do $V_{r}, I_{5}$ edo $I_{30}$ aliados ao $V_{u}$, nos parâmetros \#2, \#3 e \#4, revelou que as variáveis da chuva, além dediminuírem as correlações com as perdas de solo, também causaram uma queda no expoente $b$ da potência em questão.

\section{Erodibilidade do solo}

Os coeficientes ajustados, representativos dos fatores erodibilidade do solo (Wischmeier, 1972; Wischmeier \& Smith, 1978; Foster et al., 1982) coeficiente $b$ do modelo linear simples (\#17) e o coeficiente a do modelo não-linear (\#1) - foram positivos, indicando função crescente entre causa e efeito. Dessa forma, nas condições estudadas para Dourados, a perda desol o por erosão foi diretamente proporcional aos referidos parâmetros deerosividade. $\mathrm{O}$ fator erodibilidade $\mathrm{K}$, estimado mediante $\mathrm{o}$ parâmetro $\mathrm{a}\left(\mathrm{V}_{\mathrm{u}}\right)^{\mathrm{b}}$, foi de $0,1444 \mathrm{t} \mathrm{ha}^{-1} \mathrm{~mm}^{-1}$. Esse valor foi bastante próximo dos obtidos para Mococa $\left(0,1726 \mathrm{t} \mathrm{ha}^{-1} \mathrm{~mm}^{-1}\right)$ e Sumé $\left(0,1070 \mathrm{t} \mathrm{ha}^{-1} \mathrm{~mm}^{-1}\right)$, respectivamente, calculados para um Podzólico Vermelho-Amareloabrúptico (Carvalho, 1992) epara um Bruno Não-Cálcico vértico (Al buquerque, 1997). No entanto, tal valor foi bastante diferente dos obtidos para um Latossolo Roxo de Campinas, que foram de $1,0127 \mathrm{t} \mathrm{ha}^{-1} \mathrm{~mm}^{-1}$ (Lombardi Neto, 1979) e de $0,0472 \mathrm{t} \mathrm{ha}^{-1} \mathrm{~mm}^{-1}$ (Carvalho, 1992).

$\mathrm{O}$ fator erodibilidade $\mathrm{K}$ estimado mediante $\mathrm{O}$ parâmetro $a+b \mathrm{EI}_{30}$ foi de $0,0037 \mathrm{t}$ ha $\mathrm{h}$ ha-1 $\mathrm{MJ}^{-1} \mathrm{~mm}^{-1}$. Em relação a outros Latossolos Roxos brasileiros, esse valor foi bastantesemelhanteàquele do horizonte $B$ do estado de São Paulo, que foi de 0,0041 t ha h ha-1 $\mathrm{MJ}^{-1} \mathrm{~mm}^{-1}$ (Bertoni \& Lombardi Neto, 1990), assim como ao valor estimado para Dourados (MS), que foi de 0,0045 t ha $h \mathrm{ha}^{-1}$ MJ ${ }^{-1} \mathrm{~mm}^{-1}$ (Hernani et al., 1997).

\section{Significado das variáveis}
a, b, c, d .......... coeficientes de regressão ajustados; $A_{\text {cam }}$................
de largura);
$A_{\text {cor }}$.................. perda de solo da parcela-padrão (kg/unidade de largura);
$A_{\text {л в }} \ldots \ldots \ldots \ldots \ldots . . . . . .$. perda de solo da parcela em estudo (kg/unidade de largura);
D .................... tempo de duração da enxurrada (h);
E .................... energia cinética da chuva (MJ ha-1 $\left.\mathrm{mm}^{-1}\right)$;
$\mathrm{EI}_{30} \ldots . . . . . . . . . . .$. parâmetro de erosividade da chuva de Wischmeier \& Smith $(1958,1965)\left(\mathrm{MJ} \mathrm{mm} \mathrm{ha}^{-1} \mathrm{~h}^{-1}\right)$;
EIA ................. parâmetro de erosividade da chuva durante a ocorrência da enxurrada $\left(\mathrm{mm}^{2} \mathrm{~h}^{-1}\right)$;
I ..................... intensidade da chuva $\left(\mathrm{mm} \mathrm{h}^{-1}\right)$;
$I_{n}$.................... maior valor de intensidade média ponderado pelo tempo de 5 e, ou, de 30 min de ocorrência $\left(\mathrm{mm} \mathrm{h}^{-1}\right)$;
$\mathrm{i}_{\mu} \ldots \ldots \ldots \ldots \ldots . . . . . . .$. valor da intensidade de ordem $\mu\left(\mathrm{mm} \mathrm{h}^{-1}\right)$; ocorrida dentro do tempo de 5 e, ou, de 30 min;
$\mathrm{I}_{30} \ldots \ldots \ldots \ldots \ldots . . . . . . . . .$. intensidade máxima da chuva em $30 \mathrm{~min}$ $\left(\mathrm{mm} \mathrm{h}^{-1}\right)$;
$\mathrm{I}_{5} \ldots \ldots \ldots \ldots \ldots \ldots$ intensidade máxima da chuva em 5 min $\left(\mathrm{mm} \mathrm{h}^{-1}\right)$;
$\mathrm{K}$.................... fator erodibilidade do solo (t $\mathrm{h} \mathrm{ha} \mathrm{hm}^{-1} \mathrm{~mm}^{-2}$ t ha-1 $\mathrm{mm}^{-1}$; t ha $\mathrm{h} \mathrm{ha}^{-1} \mathrm{MJ}^{-1} \mathrm{~mm}^{-1}$ );




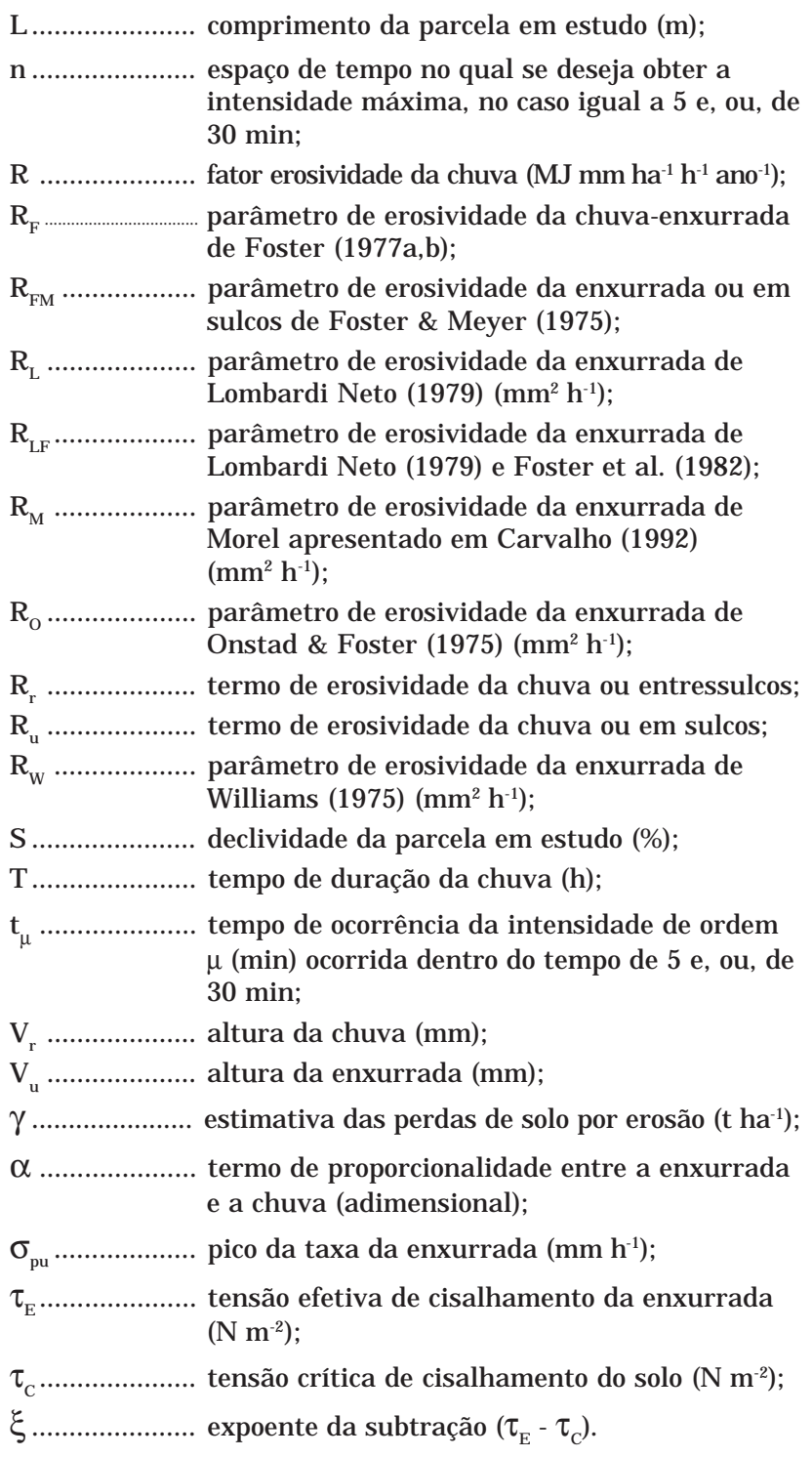

\section{CONCLUSÃO}

1. O modelo não-linear com a altura da enxurrada $\left[a\left(V_{u}\right)^{b}\right]$ foi o que melhor explicou as variações das perdas de solo das chuvas individuais $\left(R^{2}=0,9944\right)$, devendo a erosividade da chuva de Dourados ser calculada pela expressão: fator $\mathrm{R}=0,1444\left(\mathrm{~V}_{\mathrm{u}}\right)^{1,0728}$ [mm].

2. $\mathrm{O}$ fator $\mathrm{K}$, erodibilidade do solo, estimado mediante o parâmetro da enxurrada $a\left(V_{u}\right)^{b}$, foi de 0,1444 t ha-1 $\mathrm{mm}^{-1}$.

\section{LITERATURA CITADA}

ALBUQUERQUE, A.W.; CHAVES, I.B. \& VASQUES FILHO, J . Características físicas da chuva correlacionadas com as perdas de solo num Regossolo Eutrófico de Caruaru (PE). R. Bras. Ci. Solo, 18:279-283, 1994.
ALBUQUERQUE, A.W. Determinação de parâmetros para a equação universal de perda de solo nas condições de Sumé (PB). Piracicaba, Escola Superior de Agricultura "Luiz de Queiroz", 1997. 133p. (Tese de Doutorado)

ALBUQUERQUE, A.W.; LOMBARDI NETO, F.; CATANEO, A. \& SRINIVASAN, V.S. Parâmetros de erosividade da chuva e da enxurrada correlacionados com as perdas de solo de um solo Bruno Não-Cál cico Vértico em Sumé (PB). R. Bras. Ci. Solo, 22:743-749, 1998.

BERTONI, J . O espaçamento dos terraços em culturas anuais, determinados em função das perdas por erosão. Bragantia, 18:113-140, 1959.

BERTONI, J . Sistemas coletores para determinação de perdas por erosão. Bragantia, 9:147-155, 1949.

BERTONI, J . \& LOMBARDI NETO, F. Conservação do solo. São Paulo, Í cone, 1990. 355p.

BRASI L. Ministério da Agricultura e do Abastecimento. Empresa Brasileira de Pesquisa Agropecuária. Resultados de pesquisa com trigo-1988. Dourados, EMBRAPA-UEPAE, 1989. 223p.

CANTALICE, J .R.B. \& MARGOLIS, E. Características das chuvas e correlação de índices de erosividade com as perdas de solo do Agreste de Pernambuco. R. Bras. Ci. Solo, 17:275281, 1993.

CARVALHO, M.P. Fator erosividade da chuva-enxurrada para chuvas individuais do estado de São Paulo. Botucatu, Universidade Estadual Paulista, 1992. 96p. (Tese de Doutorado)

CARVALHO, M.P.; LOMBARDI NETO, F.; VASQUES FILHO, J . \& CATANEO, A. Índices de erosividade da chuva e enxurrada correlacionados com as perdas de solo e determinação da erodibilidade de um Latossolo Roxo distrófico de Campinas (SP). R. Bras. Ci. Solo, 17:445-450, 1993.

CARVALHO, M.P.; CATANEO, A. \& LOMBARDI NETO, F. Parâmetros de erosividade da chuva e da enxurrada correlacionados com as perdas de solo e determinação da erodibilidade de um Podzólico Vermelho-Amarelo de Pindorama. R. Bras. Ci. Solo, 21:279-286, 1997.

CARVALHO, M.P.; LOMBARDI NETO, F.; VASQUES, FILHO, J . \& CATANEO, A. Índices de erosividade da chuva correlacionados com as perdas de um Podzólico Vermel hoAmarelo eutrófico textura argilosa / muito argilosa de Mococa/SP: primeira aproximação do fator erodibilidade do solo. R. Bras. Ci. Solo, 13:237-242, 1989.

DRAGOUN, F.J . Rainfall energy as related to sediment yield. J . Geophys., Res., 67:1495-1501, 1962.

FOSTER, G.R.; LOMBARDI NETO, F.\& MOLDENHAUER, W.C. Evaluation of rainfall-runoff erosivity factors of individual storms. Trans. Am. Soc. Agric. Eng., 25:124-129, 1982.

FOSTER, G.R.; MCCOOL, D.K.; RENARD, K.G. \& MOLDENHAUER, W.C. Conversion of the universal soils loss equation to SI metric units. J . Soil Water Conserv., 36:355-359, 1981.

FOSTER, G.R. \& MEYER, L.D. A closed-form soil erosion equation for uplands areas. In: SHEN, H.W., ed. Sedimentation: symposium to honor professor H.A. EINSTEIN. Fort Collins, USDA, 1972. p.1-19. 
FOSTER, G.R. \& MEYER, L.D. Mathematical simulation of upland erosion by fundamental erosion mechanics. In: PRESENT AND PROSPECTIVE TECHNOLOGY FOR PREDICTING SEDIMENT YIELDS AND SOURCES, 1, Oxford, 1972. Proceedings. Oxford, United States Agricultural Research Service, 1975. p.190-207.

FOSTER, G.R.; MEYER, L.D. \& ONSTAD, C.A. An erosion equation derived from basic erosion principles. Trans. Am. Soc. Agric. Eng., 20: 678-682, 1977a.

FOSTER, G.R. \& MEYER, L.D. \& ONSTAD, C.A. A runoff erosivity factor and variable slope length exponents for soil loss estimates. Trans. Am. Soc. Agric. Eng., 20:683-687, 1977b.

GRAYBILL, F.A. Theory and application of the linear model. North Scituate, Duxbury Press, 1976. 704p.

HERNANI, L.C.; SALTON, J.C.; FABRICIO, A.C.; DEDECEK, R. \& ALVES J r., M. Perdas por erosão e rendimentos de soja e de trigo em diferentes sistemas de preparo de um solo Latossolo Roxo de Dourados (MS). R. Bras. Ci. Solo, 21:667676, 1997.

HOFFMANN, R. \& VIEIRA, S. Análise de regressão: uma introdução à E conometria. São Paulo, EDUSP, 1977. 339p.

HUDSON, N.W. Soil conservation. 2.ed. Ithaca, Cornell Universitary Press, 1973. 320p.

J ACOBS, G.A. Erodibilidade de dois L atossol os Vermel ho-E scuro (textura argilosa e média) em relação a erosividade. Curitiba, Universidade Federal do Paraná, 1995. 86p. (Tese de Mestrado)

LOMBARDI NETO, F. Rainfall erosivity - its distribution and relationship with soil loss at Campinas, Brazil. West Lafayette, PurdueUniversity, 1977. 53p. (Tesede Mestrado)

LOMBARDI NETO, F. Universal Soil Loss Equation (USLE), runoff erosivity factor, slope length, and slope steepness for individual storms. West Lafayette, Purdue University, 1979. 128p. (Tese de Doutorado)

MARQUES, J .Q.A. Determinação de perdas por erosão. Arch. Fit. Uruguai, 4:505-556, 1951.

MARQUES, J J.G.S.M.; ALVARENGA, R.C.; CURI, N.; SANTANA, D.P. \& SILVA, M.L.N. Índices de erosividade da chuva, perdas de solo e fator erodibilidade para dois sol os da região dos cerrados-primeira aproximação. R. Bras. Ci. Solo, 21:427-434, 1997.

MEYER, L.D.; FOSTER, G.R. \& ROMKENS, M.F.M. Source of soil eroded by water from upland slopes. I n: PRESENT AND PROSPECTIVE TECHNOLOGY FOR PREDICTING SEDIMENT YIELDS AND SOURCES, 1., Oxford, 1972. Proceedings. Oxford, United States Agricultural Research Service, 1975. p.177-189.
MEYER, L.D. \& WISCHEMEIER, W.H. Mathematical simulation of the process of soil by water. Trans. Am. Soc. Agric. Eng.,, 12:754-758, 1969.

MORAIS, L.F.B.; MUTTI, L.S.M. \& ELTZ, F.L.F. Índices de erosividade correlacionados com as perdas de solo no Rio Grande do Sul. R. Bras. Ci. Solo, 12: 281-284, 1988.

MUTCHLER, C.K. \& YOUNG, R.A. Soil detachment by raindrops. In: PRESENT AND PROSPECTIVE TECHNOLOGY FOR PREDICTING SEDIMENT YIELDS AND SOURCES, 1, Oxford, 1972. Proceedings. Oxford, United States Agricultural Research Service, 1975. p.113-117.

ONSTAD, C.A. \& FOSTER, G.R. Erosion modeling on watershed. Trans. Am. Soc. Agric. Eng., 19:288-292, 1975.

SILVA, M.L.N.; FREITAS, P.L.; BLANCANEAUX, P.; CURI, N. \& LIMA, J .M. Relação entre parâmetros da chuva e perdas de solo e determinação da erodibilidade de um Latossolo Vermel ho-Escuro em Goiânia (GO). R. Bras. Ci. Solo, 21:131137, 1997.

WILLIAMS, J .R. Sediment-yield prediction with universal equation using runoff energy factor. In: PRESENT AND PROSPECTIVE TECHNOLOGY FOR PREDICTING SEDIMENT YIELDS AND SOURCES, 1., Oxford, 1972. Proceedings. Oxford, United States Agricultural Research Service, 1975. p.244-252.

WILLIANS, J .R.; HILER, E.A. \& BAIRD, R.W. Predicting of sediment yields from small watersheds. Trans. Am. Soc. Agric. Eng., 14:1157-1162, 1971.

WISCHMEIER, W.H. A rainfall erosion index for a universal soil Ioss equation. Proc. Soil Sci. Am., 23:246-299, 1959.

WISCHMEIER, W.H. Upslopeerosion analyses. In: SHEN, H.W., ed. Environmental impact on rivers. Fort Collins, 1972. p.1-26.

WISCHMEIER, W.H. Use and misuse of the universal soil loss equation. J . Soil Water Conserv., 31:4-9, 1976.

WISCHMEIER, W.H. \& SMITH, D.D. Rainfall energy and its relationship to soil loss. Trans. Am. Geophys. Union, 39:285291, 1958.

WISCHMEIER, W.H. \& SMITH, D.D. Predicting rainfall erosion losses from cropland east of the Rocky Mountains: guide for selection of practices for soil and water conservation. Washington, USDA, 1965. 47p. (Agriculture handbook, 282)

WISCHMEIER, W.H. \& SMITH, D.D. Predicting rainfall erosion losses - a guide to conservation planning. Washington, USDA, 1978. 58p. (Agriculture handbook, 537)

YOUNG, R.A. \& WIERSMA, J.L. The role of rainfall impact in soil detachment and transport. Water Res. Res., 9:16291636, 1973. 